\title{
ELEMENTOS DA PAISAGEM COMO FONTE DE HETEROGENEIDADE FLORÍSTICO- ESTRUTURAL DO COMPONENTE ARBÓREO EM ÁREA DE FLORESTA OMBRÓFILA MISTA
}

\author{
LANDSCAPE ELEMENTS AS A SOURCE OF FLORISTIC-STRUCTURAL HETEROGENEITY OF \\ THE TREE COMPONENT IN AN AREA OF OMBROPHILOUS MIXED FOREST
}

\author{
Pedro Higuchi ${ }^{1}$ Ana Carolina da Silva ${ }^{1}$ Fábio Rodrigues Spiazzi ${ }^{2}$ Marcelo Negrini ${ }^{2}$ \\ Silvio Luis Rafaeli Neto ${ }^{3}$ Marco Antonio Bento ${ }^{2}$ Diego Felipe Morés ${ }^{2}$ Manoela Drews de Aguiar ${ }^{2}$ \\ Fernando Buzzi Junior ${ }^{2}$ André Leonardo da Silva²
}

\begin{abstract}
RESUMO
O presente estudo teve como objetivo testar a hipótese de que os diferentes elementos da paisagem, como os corredores, fragmentos conectados e fragmentos não conectados, apresentam o componente arbóreo com composição florístico-estrutural diferentes entre si. Para isto, o componente arbóreo de um sistema de fragmentos e corredores florestais foi amostrado por meio de 70 parcelas permanentes de $10 \times 20 \mathrm{~m}$, totalizando 1,4 ha amostrado. Dentro das parcelas, foram identificados e quantificados os indivíduos arbóreos com circunferência a altura do peito (CAP) maior ou igual a $15,7 \mathrm{~cm}$. As métricas da paisagem (área, distância do vizinho mais próximo e relação borda interior) foram extraídas de imagem LANDSAT, por meio do programa FRAGSTAT. A organização e a riqueza do componente arbóreo na paisagem foram avaliadas por meio do Escalonamento Multidimensional Não métrico (NMDS), da análise de variância multivariada não paramétrica (NPMANOVA), dos índices de dissimilaridade de Jaccard e Bray-Curtis, da rarefação, do diagrama de Venn e análise de espécies indicadoras. Foram identificados três fragmentos não conectados, dois conectados e dois corredores florestais. Neles, foram observadas 84 espécies arbóreas, pertencentes a 60 gêneros e 36 famílias. O elemento da paisagem floristicamente mais distinto foi o corredor. A hipótese de que os diferentes elementos da paisagem apresentam o componente arbóreo com composição florístico-estrutural diferentes entre si foi aceita, reforçando a ideia de que a organização espacial de fragmentos e corredores florestais são relevantes na organização da vegetação arbórea em uma escala de paisagem.
\end{abstract}

Palavras-chave: Floresta com Araucária; fragmentação florestal; ecologia da paisagem; efeito de borda.

\begin{abstract}
This study aimed to test the hypothesis that different landscape elements, such as corridors, connected and non-connected forest fragments, have a tree component with floristic-structural differences. For this sake, the tree component of a fragment-corridor system was sampled by permanent plots $(10 \times 20 \mathrm{~m})$, totaling an area of $1.4 \mathrm{ha}$. Within the plots, tree individuals with the circumference at breast height (cbh) greater than $15.7 \mathrm{~cm}$, which botanical identities were determined in the field, were sampled and counted. The landscapes metrics (fragment size area, nearest neighbor distance and edge-interior relation) were extracted from a LANDSAT image, by FRAGSTAT software. The organization and richness of the tree component

1 Engenheiro Florestal, Dr., Professor do Departamento de Engenharia Florestal, Universidade do Estado de Santa Catarina, Av. Luiz de Camões, 2090, CEP 88520-000, Lages (SC), Brasil. higuchip@gmail.com / carol_sil4@ yahoo.com.br

2 Engenheiro Florestal, Departamento de Engenharia Florestal, Universidade do Estado de Santa Catarina, Av. Luiz de Camões, 2090, CEP 88520-000, Lages (SC), Brasil.maxplus2007@yahoo.com.br / engfmarcelonegrini@ hotmail.com / marcoanbento@gmail.com / diego_fmores@hotmail.com / manooaguiar@gmail.com / buzzifjr@ hotmail.com / an_dre_sil_va@hotmail.com

3 Engenheiro Agrônomo, Dr., Professor do Departamento de Engenharia Ambiental, Universidade do Estado de Santa Catarina, Av. Luiz de Camões, 2090, CEP 88520-000, Lages (SC), Brasil. silvio.rafaeli@udesc.br
\end{abstract}

Recebido para publicação em 12/12/2012 e aceito em 10/04/2017

Ci. Fl., v. 28, n. 2, abr .- jun., 2018 
in the landscape were analyzed through Non-Metric Multidimensional Scaling (NMDS), non-parametric multivariate variance analysis (NPMANOVA), Jaccard and Bray-Curtis dissimilarities indices, rarefaction, Venn diagram and indicator species analysis. Three non-connected fragments, two connected fragments and two forest corridors were recognized. In these landscape elements, a total of 84 species, 60 genera and 36 botanical families were found. The most floristically distinctive landscape element was the corridor. The hypothesis that the different landscape elements have a tree component with floristic-structural differences was accepted, supporting the idea that the spatial organization of forest fragments and corridors is relevant for the organization of tree vegetation in a landscape scale.

Keywords: Araucaria Forest; forest fragmentation; landscape ecology; edge effect.

\section{INTRODUÇÃO}

Assim como toda a região do Domínio Atlântico, a Floresta com Araucária passou por um histórico processo de ocupação das terras, de forma que, atualmente, restam cerca de $12,6 \%$ de sua cobertura original. Este processo resultou em uma paisagem fragmentada e de estruturação diversificada, com fragmentos vegetacionais de diferentes tamanhos, formas e estágio de conservação (RIBEIRO et al., 2009). Por isto, conhecer essa estruturação da paisagem é relevante, uma vez que a organização da comunidade arbórea presente é dependente desta, devido a sua influência sobre a capacidade de dispersão e o estabelecimento das espécies arbóreas (JESUS et al., 2012). Vários estudos têm demonstrado que, dentre os elementos da paisagem com grande importância para o estabelecimento de comunidades vegetais, destacam-se os fragmentos de habitat, as matrizes de entorno e os corredores (e.g. METZGER, 1997; LAURANCE et al., 2002; CASTRO; VAN DEN BERG, 2013). Freemark, Bert e Villard (2002) definiram um fragmento de habitat como uma área relativamente homogênea que se difere da área de entorno. Para Metzger (2001), fragmentos na paisagem são "manchas" originadas a partir do processo de fragmentação de unidades vegetacionais que se apresentavam previamente contínuas. Já corredores foram definidos por Beier e Noss (1998) como habitat lineares, inseridos em uma matriz dissimilar, que conectam dois ou mais blocos maiores de habitat do mesmo tipo. Por sua vez, a matriz de entorno representa o tipo de habitat dominante na paisagem (FORMAN, 1995).

Considerando os diferentes elementos da paisagem, observa-se que a capacidade de uma espécie vegetal ocupar novas áreas pode estar relacionada com a distância da fonte de propágulo, a qualidade da matriz inter-hábitat e a síndrome de dispersão. Mesmo pequenas distâncias podem inibir a movimentação inter-hábitat de algumas espécies, resultando em isolamento espacial. Laurance e Gomez (2005), por exemplo, verificaram que clareiras com larguras superiores a $250 \mathrm{~m}$ na Amazônia representaram uma barreira para a movimentação de algumas espécies de pássaros, limitando a dispersão zoocórica. Normalmente, em ambientes fragmentados, espécies dispersas pelo vento, como, por exemplo, aquelas pertencentes à família Asteraceae, dominam quantitativamente a chuva de semente nos fragmentos florestais (JESUS et al., 2012), o que poderia resultar na predominância deste grupo. Além do isolamento descrito acima, o filtro ambiental existente e as interações ecológicas desempenham papel primordial sobre a capacidade das espécies de se estabelecerem em um fragmento florestal. Trabalhos têm demonstrado que, em ecossistemas fragmentados, a área do fragmento e o efeito de borda influenciam na qualidade e heterogeneidade ambiental existente, afetando o padrão de regeneração natural (BENITEZ-MALVIDO, 1998) e a diversidade de espécies arbóreas (HILL; CURRAN, 2003). Por exemplo, Hill e Curran (2003) verificaram que fragmentos com a forma irregular, com maior proporção de bordas, favoreceram a ocorrência de regenerantes de espécies pioneiras, devido à maior incidência lumínica. Em algumas circunstâncias, pode ser encontrada maior diversidade em áreas de borda, como observado por Nunes et al. (2003), pelo fato de que, segundo estes mesmos autores, nestas áreas, a composição florística pode ser caracterizada pela mistura de espécies típicas de interior com aquelas favorecidas pelo ambiente de borda, geralmente exigentes em luz.

No Brasil, a maior parte dos trabalhos sobre a influência da fragmentação na organização da comunidade arbórea está concentrada na região amazônica (e.g. LAURANCE; VASCONCELOS, 2009), na qual o processo de fragmentação é caracterizado pela transformação de florestas contínuas em áreas descontínuas. Estudos realizados em floresta com Araucária são menos frequentes (por exemplo, FERREIRA et al., 2016), especialmente em regiões em que esta floresta ocorre como fragmentos naturais, inseridos 
em uma matriz de campos (por exemplo, FONTOURA et al., 2006). Neste cenário, o entendimento da organização espacial de comunidades vegetais em áreas fragmentadas de floresta com Araucária assume grande importância, pois servem como subsídio para a definição de estratégias de manejo florestal e restauração de áreas degradadas. A partir de estudos dessa natureza é possível fazer inferências a respeito de configurações e arranjos espaciais mais adequados para a definição de unidades de conservação e de espécies mais indicadas para serem utilizadas em plantios de enriquecimento e, ou, adensamento em fragmentos apresentando diferentes formas e áreas.

Diante do exposto, o presente trabalho teve como objetivos: i) caracterizar as métricas da paisagem (área da classe, relação entre borda e interior e distância do vizinho mais próximo) em uma área com a presença de fragmentos florestais isolados e conectados por corredores naturais; e ii) analisar se a organização do componente arbóreo na área apresenta variações associadas ao isolamento e conectividade dos fragmentos. Desta forma, procurou-se testar a hipótese de que os diferentes elementos analisados (corredores, fragmentos conectados e fragmentos não conectados) apresentam componentes arbóreos com composição florístico-estrutural distintos entre si.

\section{MATERIAL E MÉTODOS}

O local de estudo situa-se na bacia hidrográfica do Rio Caveiras (área aproximada de $2450 \mathrm{~km}^{2}$ ), na região do Planalto Sul de Santa Catarina. O estudo foi conduzido em um sistema de fragmentos e corredores florestais, com a vegetação classificada como Floresta Ombrófila Mista Montana (IBGE, 1992), situados no município de Lages-SC, nas coordenadas $27^{\circ} 48^{\prime} 51^{\prime \prime} \mathrm{S}$ e $50^{\circ} 13^{\prime} 59^{\prime \prime} \mathrm{O}$ e altitude aproximada de $1000 \mathrm{~m}$. A partir de observações de campo, notou-se a predominância de solos classificados como Cambissolo, de acordo com o Sistema Brasileiro de Classificação de Solos (SANTOS et al., 2013).

O componente arbóreo foi inicialmente amostrado por Negrini et al. (2014), por meio de 50 parcelas permanentes de $10 \times 20 \mathrm{~m}$, distribuídas em quatro fragmentos de forma estratificada-sistemática, distantes $10 \mathrm{~m}$ entre si, considerando as suas variações ambientais, como borda e interior. Nelas, foram amostrados indivíduos arbóreos com circunferência a altura do peito (CAP) maior ou igual a 15,7 cm, cujas identidades botânicas foram reconhecidas no campo. Aqueles não reconhecidos no campo foram coletados e identificados por meio de literatura especializada e consulta a especialistas. Também foram feitos caminhamentos em todo o sistema de fragmentos e corredores para complementar a lista florística. Para este trabalho, o levantamento da vegetação foi ampliado, utilizando-se a mesma metodologia, amostrando mais um fragmento e, também, corredores florestais que conectavam os fragmentos do sistema, totalizando 70 parcelas e uma área amostral de 1,4 ha. Os fragmentos florestais foram classificados como conectados quando havia um corredor ligando-o a outro fragmento, ou isolado, quando não havia conexões. Assim, nos elementos da paisagem classificados como fragmentos isolados, fragmentos conectados e corredores, foram alocadas, respectivamente, 35, 25 e 10 parcelas. A matriz vegetacional do entorno é formada, predominantemente, por áreas com atividades agropecuárias. Para o cálculo da área de borda considerou-se a distância perpendicular da matriz florestal para o interior da floresta de até $30 \mathrm{~m}$. Esta distância de borda foi considerada de forma hipotética para o presente estudo, em função da pequena dimensão dos fragmentos avaliados e em função de que, apesar de poucos estudos sobre o tema neste tipo de fitofisionomia, há evidências que, a partir de $25 \mathrm{~m}$, ocorrem mudanças na estrutura e riqueza de plântulas de espécies arbóreas (FONTOURA et al., 2006). Ainda, assume-se que a área de borda representa uma aproximação das possíveis alterações abióticas e bióticas que caracterizam o efeito de borda. Foram considerados como corredores as áreas de florestas naturais lineares que conectavam dois fragmentos e que possuíam largura igual ou inferior a $60 \mathrm{~m}$, tendo toda sua área sob influência de borda $(30 \mathrm{~m})$. Pequenos fragmentos conectados por corredores, cujas áreas de interior foram menores do que 1,6 ha, foram considerados como parte dos corredores. Assim, a paisagem avaliada constituiu-se de três fragmentos isolados, F1, F2 e F3; e dois fragmentos conectados, F4 e F5, interligados por meio de dois corredores (Figura 1). 


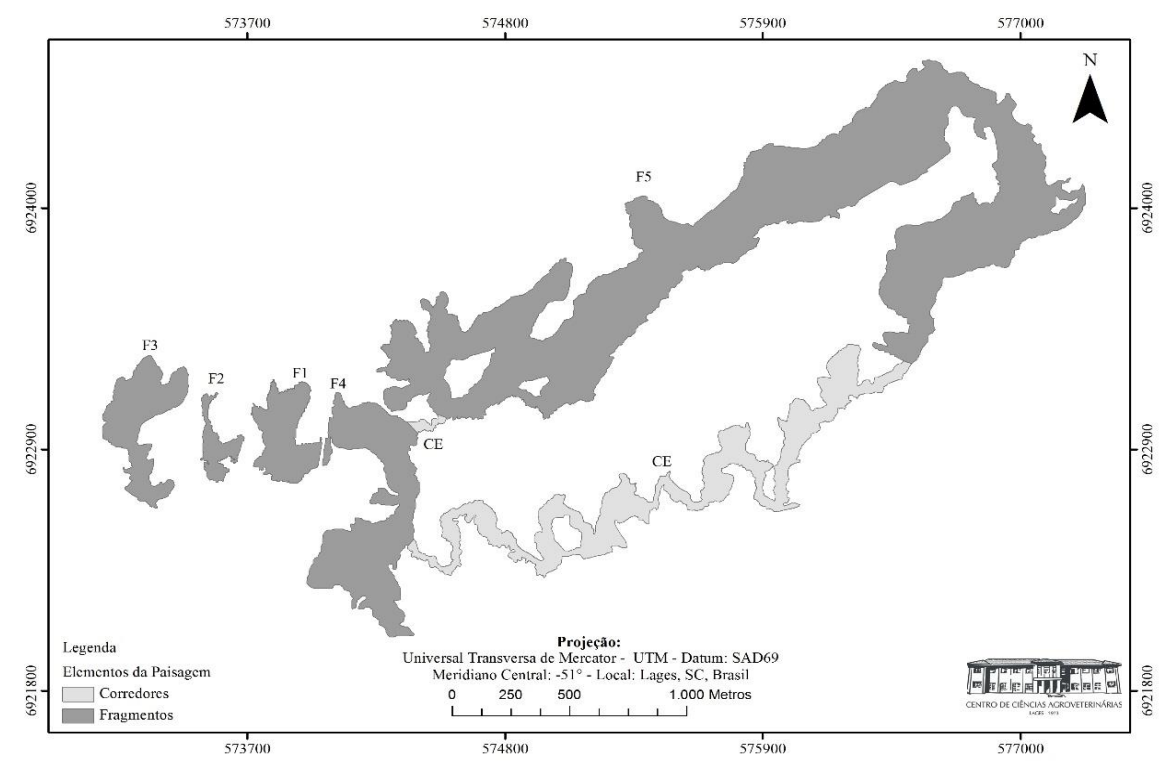

FIGURA 1: Localização geográfica dos fragmentos não conectados por corredores (F1, F2 e F3), fragmentos conectados por corredores (F4 e F5) e corredores (CE), no município de Lages, Santa Catarina.

FIGURE 1: Geographic location of non-connected fragments (F1, F2 and F3), fragments connected by corridors (F4 and F5) and corridors (CE) in the municipality of Lages, SC state.

Para a determinação das métricas de paisagem, foram utilizadas cenas do sensor TM/Landsat7 de 2007 para a produção dos mapas de cobertura do solo. As imagens foram classificadas utilizando o programa $\operatorname{ArcGis}{ }^{\circledR}$ (versão 9.3), pelo método de classificação supervisionada por máxima verossimilhança e aferida pela verdade de campo. Os pixels das imagens foram reamostrados, sendo convertidos de $10 \times$ $10 \mathrm{~m}$ para $3 \times 3 \mathrm{~m}$. As análises das métricas dos fragmentos foram conduzidas no programa FRAGSTATS (versão 3.3) (MCGARIGAL et al., 2002). As seguintes métricas foram calculadas para os fragmentos: A - área da classe (área total da classe de paisagem representada por fragmentos florestais e corredores, em ha); BI - relação borda interior para os fragmentos, considerando a área de borda (30 m); e DVP - distância do fragmento ao seu vizinho mais próximo, representada pela distância euclidiana borda a borda entre o fragmento e o fragmento mais próximo.

A suficiência amostral para a caracterização florística foi verificada por meio da curva de acumulação de espécies, por meio do método de aleatorização, com 1000 permutações. A organização do componente arbóreo na paisagem foi analisada por meio de um Escalonamento Multidimensional Não Métrico (NMDS). Foi realizada uma Análise de Variância Multivariada Não Paramétrica (NPMANOVA) para verificar a existência de diferenças significativas da estrutura do componente arbóreo presente nos diferentes elementos analisados: "fragmentos conectados", "fragmentos isolados" e "corredores". A comparação da riqueza entre os elementos da paisagem foi avaliada por meio da rarefação, considerando como base de comparação o número de indivíduos do elemento de menor abundância. Com o propósito de encontrar espécies típicas de cada elemento, foi realizada a análise de espécies indicadoras, que se baseia na abundância e frequência relativa das espécies em cada elemento da paisagem (DUFRENE; LEGENDRE, 1997). As dissimilaridades florística e estrutural entre os elementos da paisagem foram verificadas, respectivamente, por meio dos índices de dissimilaridade de Jaccard (IDJ) e de Bray-Curtis. O compartilhamento de espécies entre os elementos foi verificado por meio de um Diagrama de Venn. Estas análises foram realizadas por meio da linguagem de programação estatística R (R CORE TEAM, 2012), junto com os pacotes Vegan (OKSANEN et al., 2012), Labdsv (ROBERTS, 2012) e VennDiagram (CHEN, 2012). 


\section{RESULTADOS}

\section{Estrutura da paisagem}

Os fragmentos e corredores apresentaram diferentes áreas, que variaram de 2,92 ha (F2) até 132,15 ha (F5), com distância do vizinho mais próximo que variou entre 0 (fragmentos conectados e corredores) a 63,6 m (F2 e F3) (Tabela 1). Ressalta-se que os fragmentos F1 e F4, distantes 12,55 m entre si, eram separados por uma rodovia. O tamanho do fragmento teve relação direta com a área de borda, com o Fragmento 2, de menor tamanho, com maior relação borda interior $(12,27)$ e o Fragmento 5, de maior tamanho, com menor relação $(0,52)$.

TABELA 1: Métricas de paisagem de um sistema de fragmentos e corredores (CE) de Floresta com Araucária em Lages-SC.

TABLE 1: Landscape metrics of a system of Araucaria Forest fragments and corridors (CE) in the municipality of Lages, SC state.

\begin{tabular}{lccc}
\hline Fragmento & $\begin{array}{c}\text { Área } \\
(\text { ha) }\end{array}$ & $\begin{array}{c}\text { Distância do vizinho mais próximo } \\
(\mathrm{m})\end{array}$ & $\begin{array}{c}\text { Relação entre borda } \\
\text { e interior }\end{array}$ \\
\hline 1 & 8,39 & 12,55 & 1,17 \\
2 & 2,92 & 63,6 & 12,27 \\
3 & 11,35 & 63,6 & 1,39 \\
4 & 25,17 & 0 & 0,86 \\
5 & 132,15 & 0 & 0,52 \\
$\mathrm{CE}$ & 31,73 & 0 & 4,26 \\
\hline Total & 211,71 & - & - \\
\hline
\end{tabular}

\section{Composição Florística}

Foram observadas 84 espécies, 60 gêneros e 36 famílias (Tabela 2). A família Myrtaceae se destacou como sendo a de maior riqueza (19 espécies), seguida por Asteraceae, Lauraceae e Salicaceae, com cinco espécies cada. Myrtaceae também se destacou por apresentar o gênero de maior riqueza, Myrcia, com seis espécies. A rarefação $(\mathrm{n}=328$ indivíduos, o valor amostrado nos corredores) indicou que a riqueza entre os elementos da paisagem foi distinta, com 43 espécies nos corredores, 52,5 (erro padrão $=2,3$ ) espécies nos fragmentos conectados e 54,6 (erro padrão $=2,5$ ) espécies nos fragmentos isolados. O número de parcelas em cada elemento da paisagem (fragmentos conectados $=25$, fragmentos isolados $=35$ e corredores $=10$ ) foi suficiente para caracterizar a composição florística, uma vez que houve um aumento na riqueza menor do que 5,0\%, com o incremento de 10\% finais na intensidade amostral de cada área (Figura 2). De acordo com Kersten e Galvão (2011), esta relação de aumento da área amostral e de riqueza indica que a suficiência amostral foi alcançada. As espécies com maior abundância foram Araucaria angustifolia (Bert.) Kuntze, com 189 indivíduos, Calyptranthes concinna DC., com 163 indivíduos, e Podocarpus lambertii Klotzsch, com 136 indivíduos, todas ocorrendo em todos os elementos avaliados: corredores (CE), fragmentos conectados (FC) e fragmentos isolados (FI). 

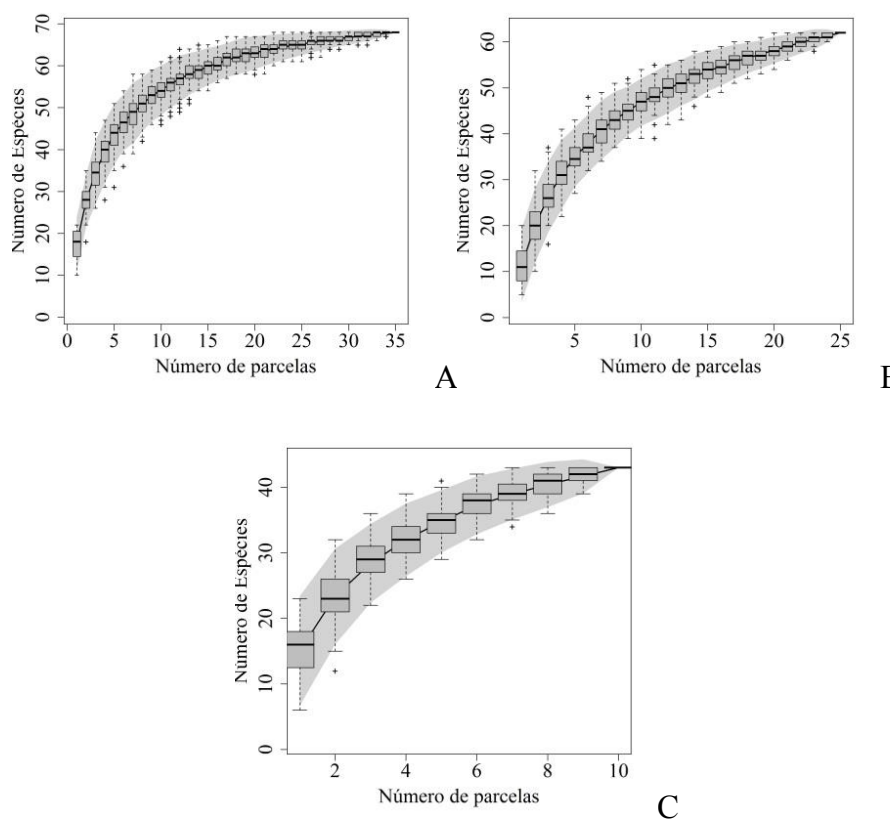

FIGURA 2: Curva de acumulação de espécies para as comunidades amostradas nos fragmentos isolados (A), conectados (B) e corredores (C), no município de Lages-SC. Gráficos do tipo de "boxplot" representam a dispersão do número de espécies de acordo com o número de parcelas.

FIGURE 2: Species accumulation curve for the tree community sampled in non-connected (A) and connected (B) fragments and corridors $(C)$ in the municipality of Lages, SC state. Boxplots represents the number of species dispersion according to number of plots.

TABELA 2: Lista florística, com a abundância de espécies em um sistema de fragmentos e corredores de Floresta Ombrófila Mista, no Município de Lages-SC. CE: corredores; FC: fragmentos conectados; FI: fragmentos isolados; T: área total.

TABLE 2: Floristic list, with abundance of species in a system of Araucaria Forest fragments and corridors, in the municipality of Lages, SC state. CE: corridors; FC: connected fragments; FI: non-connected isolated fragments; $\mathrm{T}$ : total area.

\begin{tabular}{|c|c|c|c|c|}
\hline Família/Espécie & $\mathrm{CE}$ & $\mathrm{FC}$ & FI & $T$ \\
\hline \multicolumn{5}{|l|}{ Anacardiaceae } \\
\hline Lithraea brasiliensis Marchand & 6 & 21 & 26 & 53 \\
\hline Schinus polygamus (Cav.) Cabrera & 0 & 0 & 1 & 1 \\
\hline Schinus terebinthifolius Raddi & 6 & 5 & 20 & 31 \\
\hline \multicolumn{5}{|l|}{ Annonaceae } \\
\hline Annona rugulosa (Schltdl.) H.Rainer & 0 & 2 & 22 & 24 \\
\hline \multicolumn{5}{|l|}{ Aquifoliaceae } \\
\hline Ilex dumosa Reissek & 1 & 2 & 2 & 5 \\
\hline Ilex microdonta Reissek & 0 & 0 & 3 & 3 \\
\hline Ilex paraguariensis A.St.-Hil. & 0 & 1 & 0 & 1 \\
\hline Ilex theezans Mart. ex Reissek & 15 & 10 & 23 & 48 \\
\hline \multicolumn{5}{|l|}{ Araliaceae } \\
\hline Oreopanax fulvus Marchal $^{1}$ & - & - & - & - \\
\hline \multicolumn{5}{|l|}{ Araucariaceae } \\
\hline Araucaria angustifolia (Bert.) Kuntze & 18 & 77 & 94 & 189 \\
\hline \multicolumn{5}{|l|}{ Asteraceae } \\
\hline Dasyphyllum brasiliense (Spreng.) Cabrera & 0 & 4 & 10 & 14 \\
\hline Dasyphyllum spinescens (Less.) Cabrera & 0 & 2 & 11 & 13 \\
\hline
\end{tabular}


TABELA 2: Continuação...

TABLE 2: Continued...

\begin{tabular}{|c|c|c|c|c|}
\hline Família/Espécie & $\mathrm{CE}$ & $\mathrm{FC}$ & FI & $\mathrm{T}$ \\
\hline Moquiniastrum polymorphum (Less.) G. Sancho & 2 & 8 & 11 & 21 \\
\hline Piptocarpha angustifolia Dusén & 0 & 2 & 0 & 2 \\
\hline Vernonanthura discolor (Spreng.) H.Rob. & 0 & 12 & 15 & 27 \\
\hline \multicolumn{5}{|l|}{ Berberidaceae } \\
\hline Berberis laurina Billb. ${ }^{1}$ & - & - & - & - \\
\hline \multicolumn{5}{|l|}{ Bignoniaceae } \\
\hline \multicolumn{4}{|l|}{ Canellaceae } & 54 \\
\hline \multicolumn{5}{|l|}{ Cannabaceae } \\
\hline $\begin{array}{l}\text { Celtis iguanaea (Jacq.) Sarg. } \\
\text { Cardiopteridaceae }\end{array}$ & 0 & 4 & 4 & 8 \\
\hline $\begin{array}{l}\text { Citronella gongonha (Mart.) R.A.Howard } \\
\text { Celastraceae }\end{array}$ & \multicolumn{4}{|c|}{ Celastraceae } \\
\hline $\begin{array}{l}\text { Maytenus dasyclada Mart. } \\
\text { Clethraceae }\end{array}$ & \multicolumn{3}{|c|}{ Clethraceae } & 4 \\
\hline $\begin{array}{l}\text { Clethra scabra Pers. } \\
\text { Cunoniaceae }\end{array}$ & 1 & 6 & 14 & 21 \\
\hline \multicolumn{5}{|l|}{ Dicksoniaceae } \\
\hline $\begin{array}{l}\text { Dicksonia sellowiana Hook. } \\
\text { Erythroxylaceae }\end{array}$ & \multicolumn{3}{|c|}{ Erythroxylaceae } & 35 \\
\hline $\begin{array}{l}\text { Erythroxylum deciduum A.St.-Hil. } \\
\text { Euphorbiaceae }\end{array}$ & \multicolumn{3}{|c|}{ Euphorbiaceae } & 6 \\
\hline Sapium glandulosum (L.) Morong & 0 & 4 & 5 & 9 \\
\hline Sebastiania brasiliensis Spreng. & 0 & 1 & 3 & 4 \\
\hline \multicolumn{5}{|l|}{ Fabaceae } \\
\hline Dalbergia frutescens (Vell.) Britton & 0 & 2 & 45 & 47 \\
\hline Erythrina cristagalli $\mathrm{L}^{1}{ }^{1}$ & - & - & - & - \\
\hline Inga lentiscifolia Benth. & 0 & 0 & 3 & 3 \\
\hline \multicolumn{5}{|l|}{ Lauraceae } \\
\hline Cinnamomum amoenum (Nees \& Mart.) Kosterm. & 8 & 2 & 7 & 17 \\
\hline Nectandra megapotamica (Spreng.) Mez & 0 & 1 & 0 & 1 \\
\hline Ocotea puberula (Rich.) Nees & 0 & 2 & 4 & 6 \\
\hline Ocotea pulchella Mart. & 5 & 7 & 14 & 26 \\
\hline \multicolumn{5}{|l|}{ Loganiaceae } \\
\hline $\begin{array}{l}\text { Strychnos brasiliensis (Spreng.) Mart. } \\
\text { Melastomataceae }\end{array}$ & \multicolumn{3}{|c|}{ Melastomataceae } & 1 \\
\hline \multicolumn{4}{|l|}{ Myrtaceae } & 2 \\
\hline Acca sellowiana (O.Berg) Burret & 3 & 3 & 5 & 11 \\
\hline Blepharocalyx salicifolius (Kunth) O.Berg & 14 & 7 & 12 & 33 \\
\hline Calyptranthes concinna DC. & 37 & 54 & 72 & 163 \\
\hline Campomanesia xanthocarpa O.Berg & 2 & 3 & 12 & 17 \\
\hline Eugenia pluriflora DC. & 8 & 12 & 16 & 36 \\
\hline Eugenia pyriformis Cambess. & 0 & 1 & 4 & 5 \\
\hline
\end{tabular}


TABELA 2: Continuação...

TABLE 2: Continued...

\begin{tabular}{|c|c|c|c|c|}
\hline Família/Espécie & $\mathrm{CE}$ & $\mathrm{FC}$ & FI & $\mathrm{T}$ \\
\hline Eugenia uniflora L. & 0 & 0 & 1 & 1 \\
\hline Myrceugenia euosma (O.Berg) D.Legrand & 10 & 1 & 6 & 17 \\
\hline Myrceugenia myrcioides (Cambess.) O.Berg & 1 & 4 & 8 & 13 \\
\hline Myrceugenia sp. & 3 & 0 & 0 & 3 \\
\hline Myrcia guianensis (Aubl.) DC. & 0 & 0 & 2 & 2 \\
\hline Myrcia hartwegiana (O.Berg) Kiaersk. ${ }^{1}$ & - & - & - & - \\
\hline Myrcia hatschbachii D.Legrand & 2 & 0 & 1 & 3 \\
\hline Myrcia selloi (Spreng.) N. Silveira. & 28 & 40 & 34 & 102 \\
\hline Myrcia palustris DC. & 17 & 28 & 55 & 100 \\
\hline Myrcia splendens (Sw.) DC. & 4 & 17 & 3 & 24 \\
\hline Myrcianthes gigantea (D.Legrand) D.Legrand & 0 & 0 & 1 & 1 \\
\hline Myrciaria delicatula (DC.) O.Berg & 3 & 11 & 40 & 54 \\
\hline Myrrhinium atropurpureum Schott & 3 & 3 & 8 & 14 \\
\hline \multicolumn{5}{|l|}{ Podocarpaceae } \\
\hline Podocarpus lambertii Klotzsch & 36 & 14 & 86 & 136 \\
\hline \multicolumn{5}{|l|}{ Primulaceae } \\
\hline Myrsine coriacea (Sw.) Roem. \& Schult. & 5 & 10 & 31 & 46 \\
\hline Myrsine lorentiziana DC. & 0 & 1 & 0 & 1 \\
\hline Myrsine umbellata & 0 & 5 & 1 & 6 \\
\hline \multicolumn{5}{|l|}{ Proteaceae } \\
\hline Roupala montana Aubl. & 2 & 0 & 5 & 7 \\
\hline \multicolumn{5}{|l|}{ Quillajaceae } \\
\hline & \multicolumn{2}{|c|}{ Rhamnaceae } & 1 & 2 \\
\hline Scutia buxifolia Reissek & 4 & 19 & 49 & 72 \\
\hline \multicolumn{5}{|l|}{ Rosaceae } \\
\hline Prunus myrtifolia (L.) Urb. & 3 & 3 & 8 & 14 \\
\hline \multicolumn{5}{|l|}{ Rubiaceae } \\
\hline Coutarea hexandra (Jacq.) K. Schum. & 1 & 0 & 0 & 1 \\
\hline \multicolumn{5}{|l|}{ Rutaceae } \\
\hline Zanthoxylum kleinii (R.S.Cowan) P.G.Waterman & 0 & 2 & 26 & 28 \\
\hline Zanthoxylum rhoifolium Lam. & 15 & 16 & 17 & 48 \\
\hline \multicolumn{5}{|l|}{ Salicaceae } \\
\hline Banara tomentosa Clos & 0 & 31 & 47 & 78 \\
\hline Casearia decandra Jacq. & 4 & 19 & 44 & 67 \\
\hline Casearia obliqua Spreng. & 0 & 1 & 12 & 13 \\
\hline Xylosma ciliatifolia (Clos) Eichler & 0 & 8 & 12 & 20 \\
\hline Xylosma tweediana (Clos) Eichler & 2 & 0 & 3 & 5 \\
\hline \multicolumn{5}{|l|}{ Sapindaceae } \\
\hline Allophylus edulis (A.St.-Hil., Cambess. \& A.Juss.) Radlk. & 0 & 11 & 11 & 22 \\
\hline Allophylus guaraniticus (A.St.-Hil.) Radlk. & 4 & 0 & 7 & 11 \\
\hline Cupania vernalis Cambess. & 0 & 1 & 0 & 1 \\
\hline Matayba elaeagnoides Radlk. & 0 & 41 & 49 & 90 \\
\hline \multicolumn{5}{|l|}{ Solanaceae } \\
\hline Solanum sanctaecatharinae Dunal & 0 & 0 & 2 & 2 \\
\hline Solanum sp. & 0 & 1 & 0 & 1 \\
\hline Solanum variabile Mart. & 0 & 1 & 0 & 1 \\
\hline Styracaceae & & & & \\
\hline
\end{tabular}

Continua... 
TABELA 2: Continuação...

TABLE 2: Continued...

\begin{tabular}{lrrrr}
\hline Família/Espécie & CE & FC & FI & T \\
\hline Styrax leprosus Hook. \& Arn. & 1 & 0 & 0 & 1 \\
Symplocaceae & 10 & 1 & 15 & 26 \\
Symplocos uniflora (Pohl) Benth. & & & & \\
Winteraceae & 20 & 17 & 36 & 73 \\
Drimys brasiliensis Miers & & &
\end{tabular}

Em que: 1 = Espécies encontradas somente no levantamento florístico.

\section{Organização da comunidade no sistema de fragmentos e corredores florestais}

De acordo com Análise de Variância Multivariada Não paramétrica, o componente arbóreo apresentou variação espacial na área, de forma que existiram diferenças florísticas e estruturais entre os elementos da paisagem $(\mathrm{p}=0,001)$. A posição do centroide de cada elemento (Figura 3), definido a partir da distribuição das parcelas na ordenação produzida pela NMDS (stress $=0,18$ ), demonstra que o elemento "corredores" foi o mais distinto, indicando maior diferença florístico-estrutural das parcelas deste local. Na Figura 3 também é possível observar que o elemento da paisagem com maior dispersão das parcelas na ordenação, indicada pela linha contínua conectando parcelas mais periféricas de cada grupo, foi "fragmentos conectados", sugerindo maior diversidade beta.

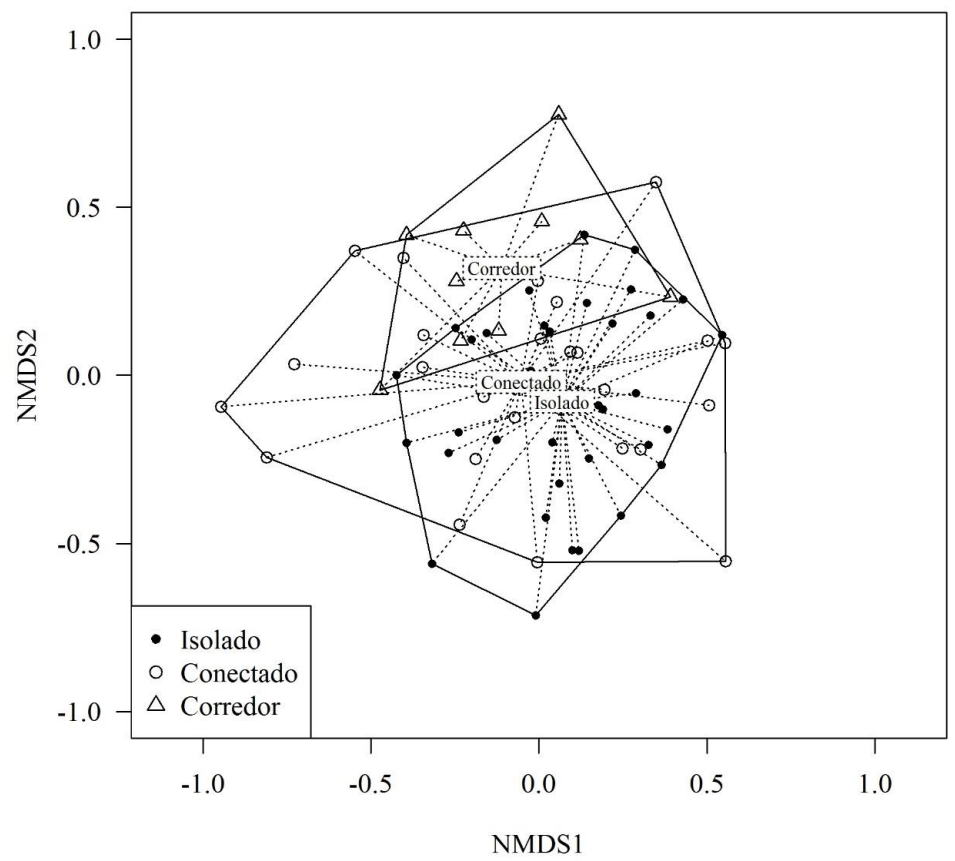

FIGURA 3: Ordenação produzida pela NMDS (stress $=0,18)$ para uma comunidade de espécies arbóreas em um sistema de fragmentos florestais (isolado: fragmentos isolados; conectado: fragmentos conectados por corredores) e corredores florestais (corredor) no Município de Lages, Santa Catarina. Os centroides (representados por retângulos com os nomes dos elementos da paisagem), indicando os valores médios dos scores das parcelas de cada elemento da paisagem, estão conectados por meio de linhas pontilhadas. Linhas contínuas estão conectando as parcelas mais externas de cada elemento da paisagem.

FIGURE 3: Ordination produced by a NMDS (stress value $=0.18$ ) for a tree species community, in a system of Araucaria Forest fragments (isolado: spatially isolated fragments; conectado: Connected Fragments by corridors) and forest corridors (Corredor), in the municipality of Lages, Santa Catarina state. The centroids (represented by rectangles with the names of landscape elements), indicating plot score average values of each landscape element, are connected by dotted lines. Continuous lines are connecting the most external plots of each landscape element. 
Foram observadas espécies indicadoras $(\mathrm{p} \leq 0,05)$ apenas para o elemento "corredores" (Blepharocalyx salicifolius (Kunth) O.Berg, Podocarpus lambertii, Drimys brasiliensis Miers, Zanthoxylum rhoifolium Lam., Cinnamomum amoenum (Nees \& Mart.) Kosterm., Symplocos uniflora (Pohl) Benth., Myrceugenia euosma (O.Berg) D.Legrand e Allophylus guaraniticus (A.St.-Hil.) Radlk.), e para "fragmentos isolados" (Cinnamodendron dinisii Schwacke, Zanthoxylum kleinii (R.S.Cowan) P.G.Waterman, Dalbergia frutescens (Vell.) Britton, Myrciaria delicatula (DC.) O.Berg e Annona rugulosa (Schltdl.) H.Rainer). Além de ser floristicamente mais distinto, o maior número de espécies indicadoras no elemento de "corredores" também pode estar relacionado com o menor número de parcelas alocadas neste ambiente, uma vez que este aspecto influencia nos valores de frequência relativa das espécies. O índice de dissimilaridade de Jaccard demonstrou menor substituição de espécies entre os "fragmentos conectados" e os "fragmentos isolados" (IDJ = 0,29), que compartilham 54 espécies entre si, o que representa 71,1\% do total que ocorreram nestes elementos da paisagem. Entre os corredores e os fragmentos ocorreu maior substituição, sendo a dissimilaridade de 0,54 entre fragmentos conectados e corredores, o que representa um compartilhamento de 33 espécies (45,8\%), e de 0,46 entre fragmentos isolados e corredores, com 39 espécies compartilhadas (54,2\%) (Figura 4). Sob o ponto de vista florístico-estrutural, a dissimilaridade observada reflete os resultados evidenciados na ordenação produzida pela NMDS, com o índice de dissimilaridade de Bray-Curtis de 0,39 entre os fragmentos isolados e conectados, 0,49 entre fragmentos conectados e corredores e 0,61 entre fragmentos isolados e corredores.

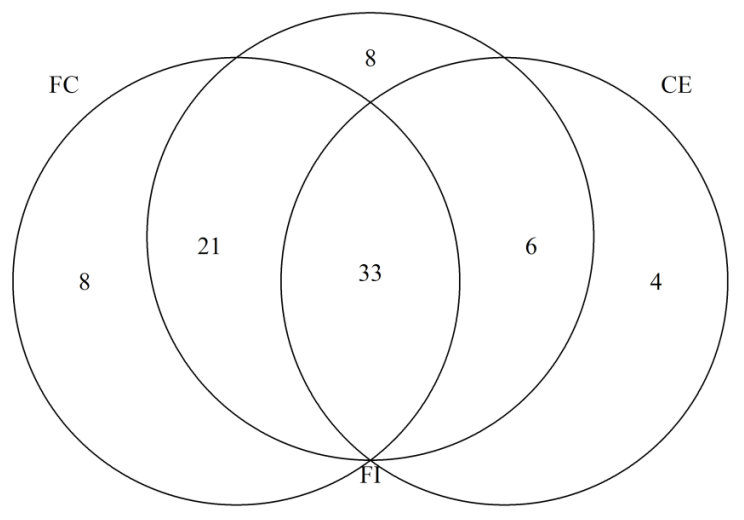

FIGURA 4: Diagrama de Venn demonstrando o compartilhamento de espécies para os diferentes elementos da paisagem estudados em um sistema de fragmentos-corredores florestais (FI: fragmentos isolados, FC: fragmentos conectados e CE: corredores) no município de Lages, Santa Catarina.

FIGURE 4: Venn diagram demonstrating species sharing among different studied landscape elements in a system of forest fragment-corridors (FI: isolated fragments, FC: connected fragments e CE: corridors), in the municipality of Lages, Santa Catarina state.

\section{DISCUSSÃO}

Considerando que a literatura existente demonstra que a estrutura da paisagem exerce forte influência sobre o ambiente abiótico e biótico (e.g. MURCIA, 1995; BENITEZ-MALVIDO, 1998; LAURANCE et al., 2002; LAURANCE; VASCONCELOS, 2009), infere-se que a heterogeneidade florístico-estrutural observada na área seja também determinada por este aspecto, como indicado pelos resultados observados. Assim, a hipótese do trabalho, de que os diferentes elementos analisados apresentam comunidades arbóreas com composição florística e estrutural diferentes entre si, foi confirmada. Em particular, os corredores apresentaram o componente arbóreo mais distinto, que foi refletido pela menor riqueza e o maior número de espécies indicadoras (Blepharocalyx salicifolius, Podocarpus lambertii, Drimys brasiliensis, Zanthoxylum 
rhoifolium, Cinnamomum amoenum, Symplocos uniflora, Myrceugenia euosma e Allophylus guaraniticus), o que sugere que este elemento da paisagem é também ambientalmente mais distinto em relação aos fragmentos analisados. Assim como no presente estudo, Castro e Van Den Berg (2013) observaram, em Floresta Estacional Semidecidual no Sul de Minas Gerais, diferenças florístico-estruturais entre fragmentos e corredores florestais, reforçando o padrão observado no presente estudo.

Com exceção do Fragmento 2 (isolado), todos os fragmentos apresentaram menor relação borda interior do que os corredores, sugerindo a maior susceptibilidade ao efeito de borda neste elemento da paisagem, o que pode ter resultado na maior distinção florístico-estrutural, como indicado na NMDS. Dentre as principais consequências da fragmentação florestal, a redução do tamanho do habitat e o surgimento e intensificação do efeito de borda (MURCIA, 1995) têm sido reportados como importantes fatores que influenciam fatores bióticos e abióticos em ecossistemas florestais, resultando em alterações na comunidade de plantas. Isso pode ter contribuído para a menor riqueza de espécies no elemento formado por corredores, já que o efeito borda pode reduzir a ocorrência de algumas espécies. É interessante observar que, dentre as espécies que não ocorreram nos corredores, algumas foram bastante abundantes nos fragmentos (mais que 50 indivíduos por espécie), como Jacaranda puberula Cham., Banara tomentosa Clos e Matayba elaeagnoides Radlk., o que leva a crer que a não ocorrência destas nos corredores não é aleatória, sugerindo a existência de alguma restrição nesse ambiente. No entanto, é importante ressaltar que o efeito de borda apresenta elevada complexidade, uma vez que está relacionado com mudanças bióticas e abióticas que ocorrem de forma não linear ao longo do espaço e do tempo (MURCIA, 1995), de forma que não se pode descartar que os resultados observados no presente estudo também estejam relacionados com outros aspectos ecológicos envolvidos, como a própria heterogeneidade ambiental existente nos elementos da paisagem avaliado.

A família Myrtaceae se destacou como a de maior riqueza, com mais da metade das espécies (12) ocorrendo nos três elementos da paisagem (FI, FC e CE). Em pesquisas realizadas em fragmentos na região (e.g. KLAUBERG et al., 2010; NASCIMENTO et al., 2011; HIGUCHI et al., 2012a; 2012b; SILVA et al., 2012; HIGUCHI et al., 2013; SILVA et al., 2013), esta família também é encontrada como sendo a de maior riqueza, demonstrando sua eficiência em ocupar fragmentos florestais. Myrtaceae também se destacou na riqueza da chuva de sementes em fragmentos no Estado de São Paulo (JESUS et al., 2012), o que demonstra sua elevada capacidade de dispersão, que pode resultar em uma ampla distribuição em paisagens fragmentadas. Araucaria angustifolia, espécie de maior abundância, também ocorreu em todos os elementos da paisagem, demonstrando que se trata de uma espécie com elevado potencial para ocorrer em fragmentos com diferentes configurações espaciais. Além disso, indivíduos de araucária podem colonizar áreas abertas na matriz campestre, atuando como elementos nucleadores de pequenos "capões" (DUARTE et al., 2006).

A baixa dissimilaridade florístico-estrutural entre os elementos da paisagem compostos por fragmentos, demonstrado pelo índice de Bray-Curtis utilizando-se uma matriz de abundância das espécies, é um indicativo de que a distância espacial produziu pouco isolamento na área estudada. Esta constatação reforça a importância dos pequenos agrupamentos de fragmentos, que estão próximos entre si $(<200 \mathrm{~m})$, de formarem mosaicos que favorecem a movimentação de espécies na paisagem (RIBEIRO et al., 2009). Esse fato, associado à elevada riqueza destes, sugere que os fragmentos isolados podem estar exercendo a função de stepping stones e são, também, importantes do ponto de vista da conservação. No entanto, a baixa dissimilaridade também pode ser o reflexo do histórico de uso da área, caracterizado pelo corte seletivo no passado e a presença de gado no interior dos fragmentos. Conforme observado por Lôbo et al. (2011) no nordeste do Brasil, este processo tende a favorecer a proliferação de grupos específicos de espécies que potencialmente podem causar a homogeneização da composição florística na paisagem. Assim, espécies supostamente mais susceptíveis ao histórico de uso podem ter sido extintas localmente, resultando em uma flora mais homogênea.

Para inferências mais conclusivas sobre os fatores que poderiam limitar a ocorrência de espécies em certos elementos da paisagem, seriam necessários estudos mais detalhados sobre a ecologia das espécies e maior detalhamento sobre as condições ambientais de cada área, abordando aspectos relacionados à dispersão de propágulos e atributos funcionais. 


\section{CONCLUSÕES}

A hipótese de que os diferentes elementos da paisagem apresentam comunidades arbóreas com composições florístico-estruturais diferentes entre si foi aceita, reforçando a ideia de que a configuração espacial de fragmentos e corredores florestais são relevantes na organização do componente arbóreo em escala de paisagem. Neste sentido, fica evidente que estratégias de conservação e manejo de fragmentos florestais, como, por exemplo, a escolha de espécies para o plantio de adensamento ou enriquecimento, devem considerar também aspectos relacionados à ecologia da paisagem. A partir dos resultados encontrados, destaca-se a maior distinção do componente arbóreo observado no elemento da paisagem formado por corredores e a baixa dissimilaridade entre fragmentos isolados e não isolados. Com isto, infere-se que pequenos fragmentos florestais isolados a pequenas distâncias, como observado no presente trabalho, são relevantes no contexto de conservação em paisagens fragmentadas, uma vez que os mesmos podem atuar como stepping stones.

\section{AGRADECIMENTOS}

Ao CNPq, pela concessão de bolsa de produtividade em pesquisa ao primeiro e ao segundo autor.

\section{REFERÊNCIAS}

BEIER, P.; NOSS, R. F. Do habitat corridors provide connectivity? Conservation Biology, Hoboken, v. 12, n. 6, p. 1241-1252, 1998.

BENITEZ-MALVIDO, J. Impact of Forest Fragmentation on Seedling Abundance in a Tropical Rain Forest. Conservation Biology, Hoboken, v. 12, n. 2, p. 380-389, abr. 1998.

CASTRO, G. C.; VAN DEN BERG, E. Structure and conservation value of high-diversity hedgerows in southeastern Brazil. Biodiversity and Conservation, Amsterdam, v. 22, n. 9, p. 2041-2056, 2013.

CHEN, H. VennDiagram: generate high-resolution Venn and Euler plots. R package version 1.5.1. [s. 1.: s. n.], 2012.

DUFRENE, M.; LEGENDRE, P. Species assemblages and indicator species: the need for a flexible asymmetrical approach. Ecological monographs, Hoboken, v. 67, n. 3, p. 345-366, 1997.

DUARTE, L. D. A. S. et al. Role of nurse plants in Araucaria Forest expansion over grassland in south Brazil. Austral Ecology, Hoboken, v. 31, n. 4, p. 520-528, 2006.

FERREIRA, T. S. et al. Composição florístico-estrutural ao longo de um gradiente de borda em fragmento de Floresta Ombrófila Mista Alto-Montana em Santa Catarina. Ciência Florestal, Santa Maria, v. 26, n. 1, p. 123-134, 2016.

FREEMARK, K.; BERT, D.; VILLARD, M. Patch-, landscape-, and regional-scale effects on biota. In: APPLYING landscape ecology in biological conservation. New York: Springer, 2002. p. 58-83.

FONTOURA, S. B. et al. Changes in plant community diversity and composition across an edge between Araucaria forest and pasture in South Brazil. Revista Brasileira de Botânica, São Paulo, v. 29, n. 1, p. 79-91, 2006.

FORMAN, R. T. T. Land mosaics: the ecology of landscapes and regions. Cambridge: Cambridge University Press, 1995.

HIGUCHI, P. et al. Floristic composition and phytogeography of the tree component of Araucaria Forest fragments in southern Brazil. Brazilian Journal of Botany, São Paulo, v. 35, n. 2, p. 145-157, 2012a.

HIGUCHI, P. et al. Influência de variáveis ambientais sobre o padrão estrutural e florístico do componente arbóreo, em um fragmento de Floresta Ombrófila Mista Montana em Lages, SC. Ciência Florestal, Santa Maria, v. 22, n. 1, p. 79-90, 2012b.

HIGUCHI, P. et al. Florística e estrutura do componente arbóreo e análise ambiental de um fragmento de Floresta Ombrófila Mista Alto-montana no município de Painel, SC. Ciência Florestal, Santa Maria, v. 23, n. 1, p. 153-164, 2013.

HILL, J. L.; CURRAN, P. J. Area, shape and isolation of tropical forest fragments: effects on tree species diversity and implications for conservation. Journal of Biogeography, Hoboken, v. 30, n. 9, p. 1391-1403, 
2003.

IBGE. Manual técnico da vegetação brasileira. 2. ed. Rio de Janeiro: Fundação Instituto Brasileiro de Geografia e Estatística, 2012. 271 p. (Manuais técnicos em geociências, n. 1).

JESUS, F. M. et al. The importance of landscape structure for seed dispersal in rain forest fragments. Journal of Vegetation Science, Hoboken, v. 23, n. 6, p. 1126-1136, 2012.

KERSTEN, R. A.; GALVÃO, F. Suficiência amostral em inventários florísticos e fitossociológicos. In: FELFILI, J. M. et al. (Org.). Fitossociologia no Brasil: métodos e estudos de casos. Viçosa, MG: Editora UFV, 2011. p. 156-173.

KLAUBERG, C. et al. Florística e estrutura de um fragmento de Floresta Ombrófila Mista no Planalto Catarinense. Biotemas, Florianópolis, v. 23, n. 1, p. 35-47, 2010.

LAURANCE, S. G. W.; GOMEZ, M. S. Clearing Width and Movements of Understory Rainforest Birds. Biotropica, Hoboken, v. 37, n. 1, p. 149-152, 2005.

LAURANCE, W. F. et al. Ecosystem decay of Amazonian forest fragments: a 22-year investigation. Conservation Biology, Hoboken, v. 16, n. 3, p. 605-618, 2002.

LAURANCE, W. F.; VASCONCELOS, H. L. Ecological consequences of forest fragmentation in the Amazon. Oecologia Brasiliensis, Rio de Janeiro, v. 13, n. 3, p. 434-451, 2009.

LÔBO, D. et al. Forest fragmentation drives Atlantic forest of northeastern Brazil to biotic homogenization.

Diversity and Distributions, Hoboken, v. 17, n. 2, p. 287-296, 2011.

MCGARIGAL, K. et al. FRAGSTATS: spatial pattern analysis program for categorical maps. 2002. Disponível em: <http://www.umass.edu/landeco/research/fragstats/fragstats.html $>$ Acesso em: 3 maio 2015.

METZGER, J. P. O que é ecologia da paisagem. Biota Neotropica, São Paulo, v. 1, n. 1/2, p. 1-9, 2001.

METZGER, J. P. Relationships between landscape structure and tree species diversity in tropical forests of South-East Brazil. Landscape and Urban Planning, Amsterdam, v. 37, n. 1, p. 29-35, 1997.

MURCIA, C. Edge effects in fragmented forests: implications for conservation. Trends in Ecology and Evolution, Amsterdam, v. 10, n. 2, p. 58-62, 1995.

NASCIMENTO, A. R. T. et al. Estrutura e classificação de um remanescente de floresta ripária no município de Lages, SC. Ciência Florestal, Santa Maria, v. 21, n. 2, p. 209-218, 2011.

NEGRINI et al. Heterogeneidade floristica-estutural do componente arbóreo em um sistema de fragmentos florestais no Planalto Sul Catarinense. Revista Árvore, Viçosa, MG, v. 38, n. 5, p. 779-786, 2014.

NUNES, Y. R. F. et al. Variações da fisionomia, diversidade e composição de guildas da comunidade arbórea em um fragmento de floresta semidecidual em Lavras, MG. Acta Botanica Brasilica, Belo Horizonte, v. 17, n. 2, p. 213-229, 2003.

OKSANEN, J. et al. Vegan: community ecology package. R package version, v.1, p. 8-8. [s. 1.: s. n.], 2012. R CORE TEAM. R: a language and environment for statistical computing. [s. 1.]: R Foundation for Statistical Computing, 2008. Disponível em: <http://www.R-project.org>. Acesso em: 18 nov. 2012.

RIBEIRO, M. C. et al. The Brazilian Atlantic Forest: how much is left, and how is the remaining forest distributed? Implications for conservation. Biological Conservation, Amsterdam, v. 142, n. 6, p. 1141$1153,2009$.

ROBERTS, D.W. Labdsv: ordination and multivariate analysis for ecology. R package version 1.5-0. [s. 1.: s. n.], 2012. Disponível em: <http://CRAN.R-project.org/package=labdsv>. Acesso em: 2012.

SANTOS, H. G. et al. Sistema brasileiro de classificação de solos. 3. ed. Brasília: EMBRAPA, 2013. 353 p.

SILVA, A. C. et al. Relações florísticas e fitossociologia de uma Floresta Ombrófila Mista Montana secundária em Lages, Santa Catarina. Ciência Florestal, Santa Maria, v. 22, n. 1, p. 193-206, 2012.

SILVA, A. C. et al. Caracterização fitossociológica e fitogegráfica de um trecho de floresta ciliar em Alfredo Wagner, SC, como subsídio para restauração ecológica. Ciência Florestal, Santa Maria, v. 23, n. 4, p. 579593, 2013. 